$10 \mid 1988$

Varia

\title{
Pascal au Japon : Le dialogue Orient-Occident
}

\section{Gérard Ferreyrolles}

\section{OpenEdition}

\section{Journals}

Édition électronique

URL : http://journals.openedition.org/ccibp/461

DOI : 10.4000/ccibp.461

ISSN : 2493-7460

\section{Éditeur}

Centre international Blaise Pascal

\section{Édition imprimée}

Date de publication : 19 septembre 1988

Pagination : 4-11

ISSN : 0249-6674

Référence électronique

Gérard Ferreyrolles, «Pascal au Japon : Le dialogue Orient-Occident », Courrier du Centre international Blaise Pascal [En ligne], 10 | 1988, mis en ligne le 07 décembre 2015, consulté le 04 mai 2019. URL : http://journals.openedition.org/ccibp/461; DOI : 10.4000/ccibp.461

Ce document a été généré automatiquement le 4 mai 2019.

Centre international Blaise Pascal 


\title{
Pascal au Japon : Le dialogue Orient- Occident
}

\author{
Gérard Ferreyrolles
}

1 En diverses occasions, monsieur J. Mesnard a rappelé avec chaleur ce qu'ont été l'agrément touristique, la découverte culturelle et l'enrichissement humain procurés aux membres de la Société des Amis de Port-Royal et du Centre International Blaise Pascal par leur séjour au Japon. Il m'incombe ici d'en évoquer l'aspect proprement intellectuel. Les congrès s'amusent, dit-on; il arrive aussi qu'ils travaillent - et ce fut le cas du nôtre. À cinq reprises, les participants se sont réunis pour écouter, réfléchir et discuter: à l'Université de Tokyo, du 27 au 29 septembre 1988; à l'Université Sophia de Tokyo, où nous avons été accueillis de façon très œcuménique par les Pères jésuites, le samedi $1^{\text {er }}$ octobre ; à l'Université municipale d'Osaka, où $\mathrm{M}$. Mesnard a prononcé une conférence, le mercredi 5 octobre ; à l'Université Kwansei-Gakuin, protestante cette fois, le vendredi 7 octobre; et enfin à la Maison des Séminaires de Sengari le samedi 8 octobre. On se bornera dans ces pages au colloque de Tokyo, mais il serait bien injuste de ne pas rappeler que toutes les autres réunions ont eu pour épine dorsale les communications de M. Jean Mesnard (il y en eut cinq, sans compter les allocutions d'ouverture et de clôture) et de M. Philippe Sellier (il y en eut trois), auxquelles il convient d'ajouter les deux conférences du Professeur Hara, spécialiste de l'histoire des mathématiques. Cette justice minimale étant rendue, revenons par la pensée dans la vaste et confortable salle Sanjo Kaïkan de l'Université de Tokyo. Contre les vitres, la pluie bat, nous prémunissant contre les tentations du vagabondage; sous nos pieds parfois la terre tremble, mais ce sont grondements d'approbation. Là vont se succéder pendant trois jours 32 conférenciers (16 Japonais, 11 Français, 2 Américains, 1 Coréenne, 1 Canadien, 1 Hollandais), dont les réflexions vont nous tenir en éveil intellectuel pendant environ 7 heures par jour. N'ayant pas été une voix pendant ce colloque, j'ai essayé d'en être l'oreille, mais il va de soi qu'avant la publication de ses Actes aucun bilan définitif ne peut en être tiré. Il est néanmoins possible, avec le secours de quelques documents et des éclairs de mémoire, de tenter une première synthèse - balbutiante et incomplète, mais susceptible tout de même de faire pressentir la très grande richesse des informations et 
propositions qui se sont alors croisées. Ce sera l'objet de cette communication sur les communications. Elle suivra 3 axes : celui des méthodes utilisées pour aborder le thème choisi, «Pascal, PortRoyal, Orient, Occident »; ensuite, celui des valeurs pascaliennes et port-royalistes qui sont communes à l'Orient et à l'Occident ; enfin, celui du dialogue des civilisations, qui définissait le « lieu » de notre rencontre en même temps que son objet.

\section{Méthodes}

2 Il est assez normal que, le colloque de Tokyo prenant après un intervalle de 12 ans la suite du colloque de Clermont-Ferrand Méthodes chez Pascal, l'on commence par se pencher sur les moyens formels utilisés pour l'approche de son sujet. Il ne s'agira plus toutefois de "méthodes chez Pascal», mais bien de "méthodes chez les pascaliens» et les portroyalisants. Or, il est frappant de constater combien à cet égard l'éventail a été ouvert, des méthodes traditionnelles aux plus récentes - sans qu'il soit naturellement question d'établir une hiérarchie entre elles, la tradition apportant son lot de certitudes, la modernité y ajoutant la stimulation de l'inédit, la gageure de montrer dans ce que tout le monde avait sous les yeux ce que personne n'avait su y voir. Le Colloque de Tokyo a débuté sous l'inspiration de Clio : qu'il s'agisse de la situation de la Pologne (décrite par François Boulétreau à travers la correspondance de Louise-Marie de Gonzague) ou de la nomination des vicaires apostoliques en Chine (examinée par M. Schmitz du Moulin à travers la correspondance de Du Vaucel), nous touchons des faits historiques; même si leur objectivité est filtrée par la subjectivité des épistoliers, c'est du moins objectivement que nous est restituée cette subjectivité, qui devient ainsi à son tour une composante de l'histoire dont elle était le témoin. Dès le commencement du colloque, la communication de F. Boulétreau a d'ailleurs soulevé un problème capital pour la façon d'aborder le thème Orient-Occident : que faut-il entendre par Orient? La Pologne peut-elle être considérée comme faisant partie de l'Orient? L'Extrême-Orient, de son côté, est-il à l'extrémité de l'Orient ou est-il extrêmement oriental ? Dans un cas, il est aux marches de l'Orient, dans l'autre il est l'Orient de l'Orient, c'est-à-dire l'Orient par excellence. Par rapport au pays du Soleil Levant, la Pologne apparaît nôtre : mais c'est qu'au mouvement de la terre s'est ajouté celui de l'histoire qui l'a fait passer, entre les $\mathrm{XVII}^{\mathrm{e}}$ et $\mathrm{XX}^{\mathrm{e}}$ siècles, d'Orient en Occident.

3 À côté de l'approche purement historique de notre thème, il y eut place, bien entendu, pour l'histoire littéraire, dans une perspective plutôt diachronique lorsqu'on situa la condamnation du théâtre par Port-Royal à l'intérieur de la tradition rigoriste. $\mathrm{M}^{\mathrm{me}}$ Goyet a montré que les théologiens et moralistes français avaient fait preuve à l'égard du théâtre d'une dureté exceptionnelle dans l'Europe de la Contre-Réforme, mais qu'il ne fallait pas attribuer aux doctrinaires de Port-Royal un fixisme inintelligent, rappelant entre autres que le fameux fragment 764 (éd. Lafuma), 630 (éd. Sellier) des Pensées sur les dangers de la comédie n'est pas de Pascal et que ce même Pascal reconnaissait, dans les Provinciales, que toutes les sévérités de la primitive Église en matière disciplinaire ne devaient point être gardées. Dans la perspective du comparatisme maintenant, nous avons pu mesurer à la fois la proximité et l'écart entre Pascal et Diderot (c'était l'exposé sur cassette de M. Nakagawa), entre Claudel et Pascal (c'était l'exposé en direct de M. Blanc). Le paradoxe résidait, pour le premier exposé, dans le rapprochement entre un janséniste et un athée; et, pour le second, dans la distance séparant un catholique d'un autre catholique : Claudel admire en Pascal le styliste, mais pour la religion, il ne concède 
à l'Apologie qu'une valeur d'usage externe : Pascal est utile pour amener les mécréants au seuil de la foi, mais à l'intérieur du temple il doit s'effacer, notamment devant saint Thomas. L'univers claudélien est un univers thomiste, c'est-àdire fini, centré et plein, saturé de sens, à l'opposé de l'univers muet, éclaté, privé de finalité parce qu'infini, auquel les Pensées confrontent leur lecteur.

Une séance du colloque

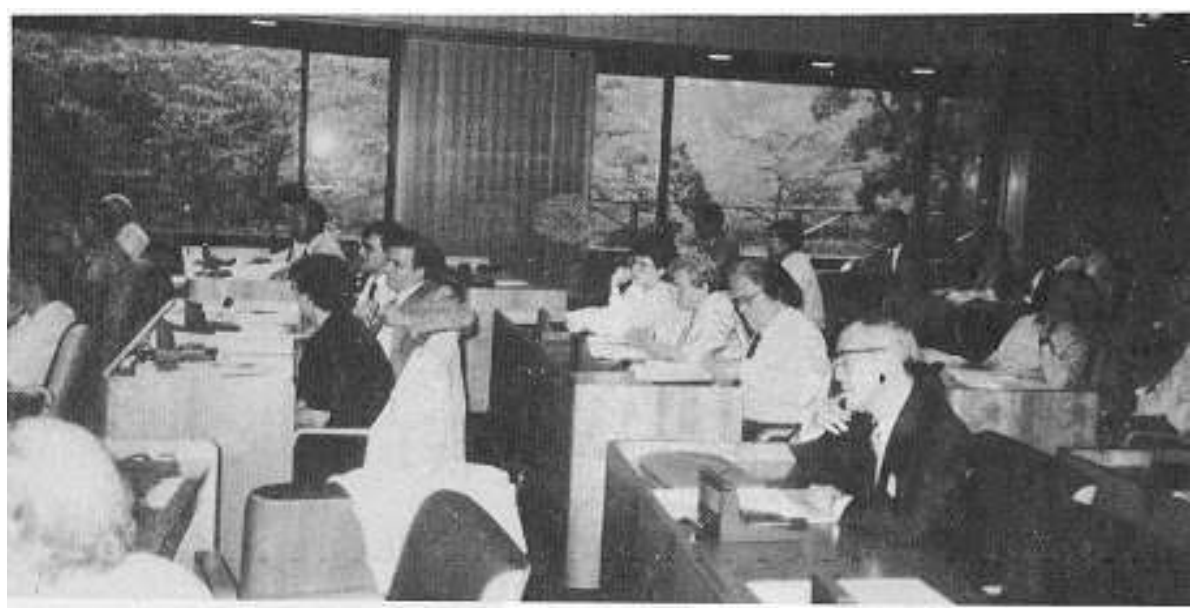

Cliché Takaharu Hasekura

Les méthodes récemment apparues dans l'histoire de la critique ont également été mises en œuvre lors du Colloque de Tokyo, et cela dans trois directions: anthropologique, herméneutique et narratologique. L'anthropologie était représentée par M. Odagiri, dont la communication sur Montherlant et le mythe de Port-Royal était sous-tendue par l'analyse girardienne du mythe et de la tragédie. On sait que, dans la conception révolutionnaire de R. Girard, exposée particulièrement dans La Violence et le Sacré et Des Choses cachées depuis la fondation du monde, le mythe et la tragédie mettent en scène le mécanisme fondateur des institutions et de la culture humaine, à savoir le sacrifice d'un innocent pris comme bouc émissaire. Pierre Force pour sa part, jeune et brillant normalien débarqué de Columbia, apportait dans ses bagages la théorie herméneutique du philosophe allemand Gadamer, grâce à laquelle il put réhabiliter la partie des Pensées considérée habituellement comme la moins défendable: celle qui contient l'argument prophétique. Contrairement au rationalisme inauguré par Descartes (et dont les origines sont peut-être à chercher du côté de la Réforme), qui juge irrecevable l'interprétation traditionnelle de l'Écriture et son utilisation pour prouver la vérité de la religion, l'herméneutique contemporaine pose que la tradition, même fautive, est la médiation nécessaire pour comprendre les textes ; comme le dit Gadamer, la communication a lieu non pas malgré les préjugés, mais grâce aux préjugés. En troisième lieu, la méthode narrotologique, dont le propos est notamment de déterminer qui parle dans un texte, a été illustrée par MM. Suematsu et Harrington. Les Pensées sont apparues comme une polyphonie, où s'entremêlent une voix non-personnelle - celle qui dit «ils » en parlant des hommes («ils n'ont que la fantaisie») -, une voix multipersonnelle - celle qui dit " nous " (l'écrivain alors s'identifie aux hommes qu'il décrit : " nous sommes incapables de vrai et de bien ») -, une voix personnelle enfin, qui est tantôt celle de l'apologiste («je vous dis que vous y gagnerez en cette vie»), tantôt celle de l'interlocuteur fictif qu'il suscite («je ne vois partout qu'obscurités»). Ces différentes instances peuvent être 
fructueusement rapprochées - comme l'a fait M. Harrington -, de celles que l'on trouve dans les Provinciales où il faut distinguer Pascal de Montalte, Montalte - personnage (celui qui parle au jésuite fictif), et deux types d'interlocuteurs (fictifs comme le Provincial ou le bon Père ; réel comme le P. Annat).

5 Au milieu de ces approches critiques abstraites et sévères, $\mathrm{M}^{\text {me }}$ Picquenard nous a apporté le rafraîchissement d'une communication audio-visuelle, dont l'exigence intellectuelle n'était pas moindre, puisqu'il s'agissait de suivre la transposition de la vie intérieure dans l'espace extérieur du tableau, en particulier chez Le Caravage, La Tour et Philippe de Champaigne.

On peut ainsi mesurer la variété et, je l'espère, la rigueur des méthodes utilisées par les participants du Colloque. Mais ces méthodes n'ont pas leur fin en elles-mêmes : elles sont au service d'une meilleure intelligence de l'œuvre pascalienne et de l'esprit de Port-Royal en général, et précisément dans le cadre de notre rencontre occidentalo-niponne, elles sont au service d'une meilleure intelligence des valeurs qui peuvent être communes à Pascal ou Port-Royal et à l'Orient.

Le buffet de I'Université de Tokyo, le 28 septembre

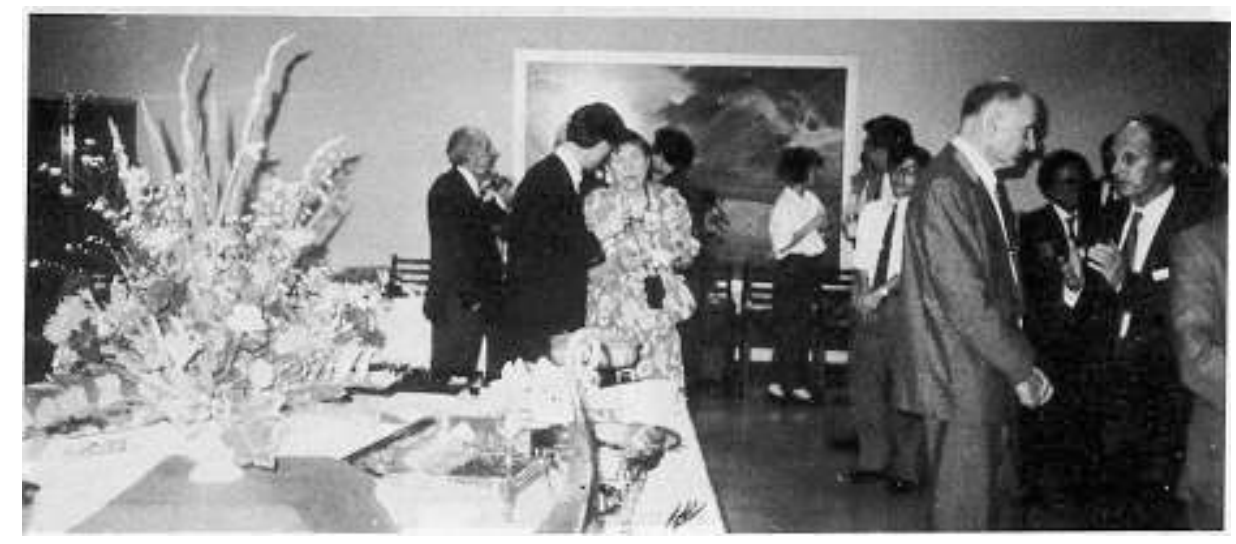

Cliché Takahura Hasekura.

\section{Valeurs}

7 Il faut recourir ici à la distinction posée par J. Mesnard dans sa communication terminale entre valeur absolue et valeurs relatives. C'est une distinction qui, à propos de Pascal, ne va pas de soi : naguère encore, la critique goldmannienne répétait que dans le jansénisme pascalien tout ce qui n'était pas valeur absolue était absolument dénué de valeur. Affirmer l'existence de valeurs relatives, c'est reconnaître un "entre-deux" où se manifeste aussi la grandeur de l'homme, cette grandeur dont M. Hirota a souligné qu'on ne devait pas la sous-estimer dans l'anthropologie pascalienne au profit d'une vision excessivement pessimiste de notre misère. Parmi les valeurs relatives, trois ont polarisé l'attention des congressistes : la science, l'honnêteté, la paix.

8 Nos amis japonais sont depuis plusieurs années des observateurs minutieux de l'œuvre scientifique de Pascal. En mathématiques, M. Hara, qui s'est penché dans un exposé d'une haute technicité sur un passage obscur du pari, a le mérite singulier d'avoir re-parcouru les démonstrations pascaliennes sans faire appel aux modernes techniques opératoires, mais en utilisant uniquement les instruments conceptuels des savants du XVII ${ }^{e}$ siècle. En 
physique, $\mathrm{M}^{\mathrm{me}}$ Koyanagi a reproduit récemment l'expérience du vide dans le vide selon le dispositif même de Pascal. Elle a proposé à Tokyo, sur des arguments très circonstanciés, d'avancer de trois ans la date de rédaction du Traité du vide (qui passerait ainsi de 1651 à 1648), ainsi que celle des Traités de l'équilibre des liqueurs et de la pesanteur de la masse de l'air (qui passerait de 1654 à 1651). Toujours sur la question du vide, M. Akagi a détaillé les étapes par lesquelles Pascal était passé de l'hypothèse d'une horreur limitée de la nature pour le vide à une explication fondée sur la seule pression atmosphérique. Un unique français, M. Allard, a mêlé ici sa voix aux communicants japonais: ce fut pour reconstituer de façon convaincante la démarche qui permit à l'adolescent Pascal de démontrer la trente-deuxième proposition d'Euclide. Signalons que cette étude est, à notre connaissance, la seule qui ait jamais été consacrée à ce sujet pourtant important, puisqu'à son occasion se révéla le génie mathématique de Blaise.

Mais, au-dessus de la science, qui ne regarde que les choses extérieures, Pascal place l'art des relations inter-humaines - l'honnêteté. Nous tenons là un trait commun à la conception pascalienne et à la conception orientale de la civilité, qui pourraient se résumer l'une et l'autre dans la formule de L. Thirouin : « un idéal lisse de la vie sociale ». Les grandeurs d'établissement sont rituellement respectées sans que jamais affleure la critique ou la contestation, dont les manifestations sont renvoyées à une demi-habileté synonyme de sottise et de bassesse d'esprit. L'honnêteté pascalienne et la civilité japonaise se rencontrent dans l'observation des bienséances. La recherche de la mesure, du naturel dans l'artificiel. La différence interviendra peut-être lorsqu'on passera du for extérieur au for intérieur : la civilité orientale est assentiment individuel à l'universalité. La première plie l'extérieur, la seconde suppose l'acquisition volontaire et réfléchie de qualités intérieures (en particulier l'oubli de soi). La civilité orientale peut facilement, selon M. Ohtomo, glisser au conformisme, mais l'honnêteté, dans la soumission apparente, garde une réserve critique - ce que Pascal appelle «la pensée de derrière ", inconnue à la mentalité japonaise. L'occidental démystifie le pouvoir, le japonais le divinise. Remarquons cependant qu'avec ce dédoublement, cette «schizophrénie sociale » que vit le pascalien (pour reprendre une expression de L. Thirouin), on n'est pas très loin de la morale bouddhiste évoquée par $\mathrm{M}$. Ohtomo encore, et qui fait dériver l'obéissance au jeu social de la conscience prise de sa futilité : comment accorder à la vanité des apparences autre chose qu'une apparence de respect? Dans la mouvance augustinienne, ce n'est pas seulement l'honnêteté qui est réhabilitée comme valeur relative, mais aussi l'honneur, voire l'héroïsme. À l'encontre de la conception un peu trop simpliste qui voyait dans l'augustinisme une machine à démolir les valeurs humaines, $\mathrm{M}$. Lafond a fort justement fait remarquer que la critique des vertus chez La Rochefoucauld laisse subsister la possibilité d'un véritable héroïsme, couronnement éblouissant d'une morale de l'honneur: les maximes les plus personnelles de La Rochefoucauld font décidément apparaître la valeur de la valeur.

$\mathrm{Au}$ sommet des valeurs relatives brille celle qui commence de réaliser pour une communauté entière l'idéal d'honnêteté poursuivi par quelques-uns de ses membres : la paix. Alors que le contraire de l'honnêteté est sottise et bassesse, qui sont des maux tolérables, le contraire de la paix signifie l'insupportable mise à mort de la société par elle-même, c'est-à-dire le plus grand des maux. Voilà pourquoi la paix est appelée par Pascal, dans la liasse «Raisons des effets », «le souverain bien » (fr. 81-116). Pourquoi alors ne pas en faire une valeur absolue ? La communication de Tetsuya Shiokawa, qui fut à coup sûr l'un des temps forts de notre colloque, apporte la réponse : la paix n'est le 
souverain bien qu'aux yeux des habiles, et encore à l'intérieur de la collectivité nationale. Dans les relations internationales, la guerre peut être juste et nécessaire. De façon analogue à l'intérieur de chaque homme, il n'y a rien de pire que de rester en paix dans ses vices: la guerre que chacun mène contre soi est, aux yeux des chrétiens, une vraie paix devant Dieu. Et ce qui vaut pour la vie spirituelle de chaque croyant vaut aussi pour l'Église «militante»: les Provinciales déclarent la guerre à la casuistique relâchée; la guerre et la paix dans l'Église ne sont que des moyens au service de la vérité, non des fins en soi. D'où le paradoxe : la guerre est légitimée dans l'Église (parce qu'elle possède la vérité et qu'il faut la défendre), mais récusée dans la cité terrestre, où le statu quo vaut mieux car on n'a rien de meilleur par quoi le remplacer.

11 La paix ne représente donc, si l'on ose dire, qu'un souverain bien relatif; le Souverain Bien au sens de valeur absolue, le Souverain Bien avec deux majuscules, c'est le «bien universel » décrit à la liasse X des Pensées, un bien tel que tous puissent le posséder « à la fois sans diminution et sans envie », en un mot Dieu lui-même. La valeur absolue, c'est donc l'Absolu comme valeur, c'est-à-dire l'Absolu comme objet de notre désir, terme de notre recherche et cause de notre béatitude. Passionnante confrontation que celle de la spiritualité orientale, spécialement bouddhiste, avec la spiritualité chrétienne, spécialement augustinienne, du XVII ${ }^{e}$ siècle. Geneviève Descamps, dans une méditation où chacun a senti combien elle s'impliquait personnellement, a mis en regard de façon suggestive des textes de Pascal et des textes décrivant la démarche Zen : ici et là sont maniés l'humour, l'ironie, le paradoxe qui plonge le lecteur ou l'auditeur dans une perplexité salutaire parce qu'il l'oblige à prendre conscience de ses limitations et à faire effort pour les dépasser. Un thème apparaît central dans cette confrontation, celui du vide. Il peut être entendu en deux sens: d'abord comme l'acte par lequel le moi se déprend de lui-même. Telle est la spiritualité de la kénose, évidente dans les Chapelets de Port-Royal, celui de la sœur Geneviève Le Tardif et celui de la Mère Agnès, qui s'alimentent au Bref Discours de l'abnégation intérieure de Bérulle, lui-même inspiré directement, comme l'a prouvé $\mathrm{M}$. Kawamata, du Breve compendio intorno alla Perfettione de la milanaise Isabella Berinzaga à la fin du XVI ${ }^{e}$ siècle. Les moines bouddhistes tendent aussi de leur part à l'anéantissement du moi ; et M. Kawamata nous a appris que ceux-ci pratiquaient autrefois l'exercice appelé "Shikan", qui consistait à s'asseoir devant un cadavre humain et à contempler des jours durant le spectacle de sa putréfaction. Mais le vide peut aussi désigner l'état où l'on parvient par l'annihilation de son moi, et là des divergences se font jour: le vide est conçu par les bouddhistes comme le but même de leur quête, un état terminal d'indifférenciation, alors que la Nuit des mystiques occidentaux n'est qu'un moment purificateur au-delà duquel leur est donnée la lumière du Christ. On voit par là les chances, mais aussi les difficultés, d'une démarche œcuménique entre chrétiens et bouddhistes. $\mathrm{M}^{\mathrm{me}}$ Weaver-Laporte a souligné le rôle important joué sur ce plan par Thomas Merton, le trappiste américain auteur de la Nuit privée d'étoiles, mort à Bangkok en 1968 lors d'une rencontre inter-monastique. Le P. Merton reconnaissait dans le bouddhisme la conscience de l'insuffisance radicale du monde phénoménal, la volonté de dépasser l'égoïsme et de tendre à l'illumination ; mais le chrétien ne peut accepter ni de considérer la personne comme une illusion ni d'exténuer la transcendance divine. Malgré un intérêt certain pour les spiritualités orientales (intérêt qui semble parfois plus marqué en occident qu'en orient) notre colloque a su garder l'esprit critique et ne pas pécher par excès de Zen. 
12 Dans l'appréciation de la ressemblance et de la différence des valeurs occidentales et orientales, une au moins était parfaitement partagée, qui a permis la discussion sur toutes les autres, c'est le sens du dialogue.

Kobé, le 6 octobre

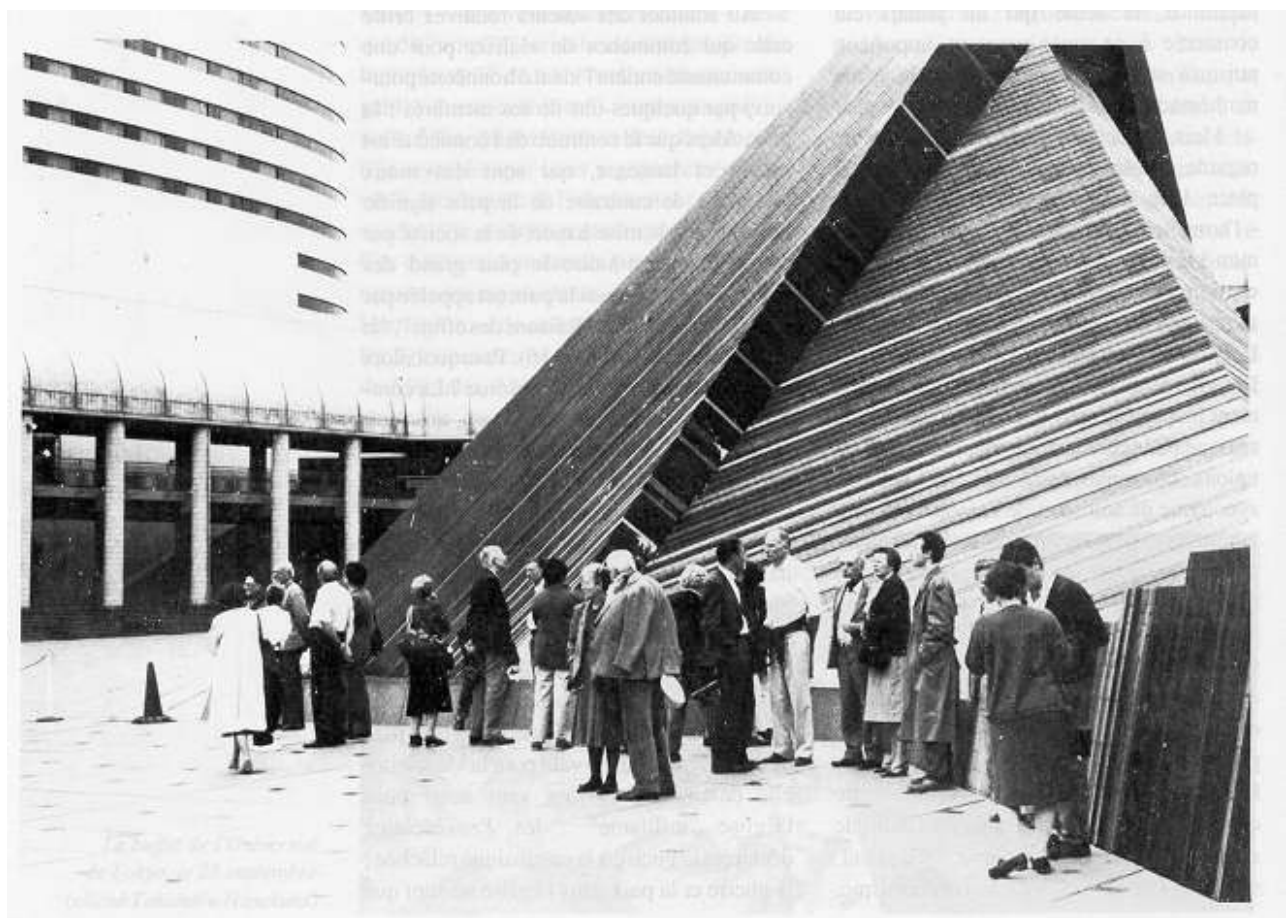

Cliché Jean Lafond

\section{Dialogue}

13 Pour qu'il y ait dialogue il faut être au moins deux, et que chacun des partenaires s'intéresse à l'autre. À priori, un handicap de conséquence grevait notre rencontre: Pascal ne s'intéresse guère au Japon. Il n'en parle qu'une fois, dans une lettre-circulaire du 7 octobre 1658 où il réfute les prétentions des mathématiciens étrangers qui demandaient un délai supplémentaire (en raison de l'éloignement) pour répondre aux questions sur la cycloïde : Pascal estime qu'alors personne ne pourrait être sûr d'avoir remporté le prix, puisque chaque jour pourraient arriver des solutions venues « du fond de la Moscovie, de la Tartarie, de la Cochinchine ou du Japon ». Le Japon n'est même pas mentionné ici pour lui-même mais à titre de symbole de l'éloignement extrême : le Japon pour Pascal est moins un pays qu'une limite. Dans son examen des religions nonchrétiennes, Pascal parle souvent de l'Islam, mais point du bouddhisme; $\mathrm{M}$. Yoichi Maeda, grand maître des études pascaliennes au Japon décédé en novembre 1987, mais rendu présent à notre colloque par la ferveur de ses disciples, trouvait que Montaigne et Malebranche allaient plus loin que Pascal dans l'ouverture sur l'Orient, et il relevait dans son Commentaire en trois volumes des Pensées (dont M. Hasekura nous a traduit plusieurs passages avec une émotion poignante) divers traits d'ethnocentrisme spontané, où la terre était réduite aux dimensions du monde méditerranéen : au fragment 1-37: "Les psaumes chantés par toute la terre », ou au fragment 413-32: «e nez de Cléopâtre, s'il eût été plus court, toute la face de la terre aurait changé ». Les pascaliens 
japonais estiment, avec quelque apparence de raison, que si le nez de Cléopâtre eût été plus court, le sort de leurs ancêtres n'en aurait pas été notablement modifié. M. Maeda remarquait au passage que l'insistance sur l'aspect court ou long du nez de Cléopâtre ne pouvait rien dire aux Japonais, dont l'unique critère de beauté en matière d'appendice nasal est sa forme basse ou retroussée. Il ajoutait un commentaire analogue au fragment 38-72: «Trop et trop peu de vin. Ne lui en donnez pas, il ne peut trouver la vérité. Donnez-lui en trop, de même ». Des buveurs de thé admettront facilement que celui qui boit trop de vin ne puisse trouver la vérité, mais ils ne conviendront certainement pas que l'abstinence de vin empêche de la découvrir.

Ces exemples plaisants montrent que la distance entre nos civilisations oblige à une conversion du regard l'oriental qui entend pénétrer le message pascalien. La distance cependant n'est pas infranchissable, et Pascal, en un sens, s'emploie à la combler. Plutôt que de se livrer à la critique facile qui consisterait à reprocher à Pascal d'avoir partagé les méconnaissances et les préjugés de son époque relatifs à l'Orient, M. Sasaki s'est plu à souligner - à propos du Proche-Orient - la possibilité d'une influence islamique dans l'argumentation du pari, et l'analogie du projet pascalien avec la démarche apologétique du théologien musulman Al-Ghazâli (XI $-\mathrm{XII}^{\mathrm{e}} \mathrm{s}$.), auteur d'un traité sur l'Incohérence de la philosophie auquel répondit Averroès. L'intérêt de Pascal pour l'Orient, et pour l'ExtrêmeOrient, s'est manifesté en tout cas de façon certaine dans ses fragments sur la Chine. Celle-ci est désignée neuf fois dans les Pensées de $\mathrm{M}$. Heller - récent auteur avec $\mathrm{M}^{\mathrm{me}}$ Goyet d'une bibliographie pascalienne - a recouru aux Sinicae Historiae du P. Martini pour éclairer les passages où il est dit que «la Chine obscurcit »: l'antiquité des dynasties chinoises venait en effet troubler les chronologistes chrétiens pour qui la Bible était un reportage infaillible sur l'histoire du monde et les Hébreux, le peuple le plus ancien de la terre. Puisqu'il est essentiel à la vérité d'être contestée par des témoignages contraires, l'objection chinoise devait finalement apporter de l'eau au moulin apologétique.

Mais Pascal eût accordé plus qu'une utilité négative à la Chine, s'il avait entendu la communication de $\mathrm{M}^{\text {lle }}$ Myung-Ai Cho, où celle-ci décelait entre la dialectique de Pascal et la dialectique de Lao-Tseu une homologie de fonctionnement d'autant plus étonnante qu'elle ne saurait s'expliquer par aucune influence de l'un de ces auteurs sur l'autre. Pascal et Lao-Tseu partent du constat de l'ambivalence au cœur de l'homme et du monde. La grandeur, la vérité, le bien coexistent avec la misère, l'erreur et le mal ; mais cette coexistence n'est pas celle, statique, de deux séries parallèles : les contraires sont relatifs l'un à l'autre, se déduisent l'un de l'autre (la grandeur se conclut de la misère et la misère de la grandeur) ; chacun des deux contraires contient son opposé (le Yin contient un Yang caché et vice-versa). Cette ambivalence est le moteur du changement: chaque élément croît jusqu'à son apogée, puis décline et révèle progressivement son opposé (suivre une vertu jusqu'à son extrémité, pour Pascal, c'est insensiblement la perdre au sein du vice). Ainsi, dans le schéma taoïste que $\mathrm{M}^{\mathrm{lle}}$ Cho a projeté sur écran, la partie noire du cercle contient un point blanc qui grossit jusqu'à recouvrir la partie noire, réduite à son tour à un point. Puisque toutes les situations humaines sont réversibles, Pascal et LaoTseu concluent ensemble que la sagesse consiste à ne pas s'abandonner tout entier à la joie devant le bonheur ou à la tristesse devant le malheur : il faut se tenir au milieu. N'allons pas plus loin dans le syncrétisme, car la conception du devenir est radicalement différente chez les deux penseurs : il est sans chute et sans avenir dans le taoïsme. La répétition éternelle du même marque chez Lao-Tseu un dieu impersonnel, tandis que la 
pensée de l'origine et de la Parousie supposent évidemment chez Pascal un Dieu personnel.

Malgré cette conception différente quant à l'essence de la divinité, la convergence est remarquable sur la nécessité pour l'homme de tendre à l'union avec la divinité. Et nous entrevoyons par là ce qui a rendu possible le dialogue Orient-Occident: les manières d'appréhender et de réaliser une valeur diffèrent selon les lieux et les temps, mais la désignation des valeurs essentielles et l'impératif de leur poursuite apparaissent communs aux civilisations les plus éloignées. Or, les Pensées sont particulièrement aptes à faire saisir l'unité de la condition humaine à travers les diverses conditions des hommes. La description pascalienne de notre misère rend sensible à $M$. Hasekura «l'unité de la nature humaine et l'identité de tous les hommes sur notre planète", cependant que $\mathrm{M}$. Mesnard montre cette unité dans la description pascalienne de notre grandeur: certes, les lois changent d'un pays à l'autre, mais il y a partout des lois et elles traduisent dans leurs imperfections même l'aspiration générale des hommes à la justice, mieux : elles manifestent l'universalité de l'Idée (au sens platonicien) de Justice; les hommes errent tous plus ou moins loin de la raison, de la vérité et du bonheur: mais tous ne veulent obéir qu'à la raison et à la vérité, et tous, mêmceux qui vont se pendre, "recherchent d'être heureux " (fr. 148-181). Tous, à travers la multiplicité parfois caricaturale des figures, visent le même archétype.

Pour que le dialogue, rendu possible par cette communauté de principes, devienne réel, il a fallu le concours des circonstances et des talents. II a fallu qu'un protestant japonais, Yûki Koh - dont la personnalité attachante a été dépeinte par M. Morikawa - découvre dans Saint-Cyran et dans Pascal un écho de sa propre interrogation spirituelle et décide, après bien des scrupules vaincus, de donner à ses compatriotes la première traduction des Pensées en japonais. L'exemple a été suivi et nous avons pu entendre le plus récent traducteur des œuvres de Pascal, M. Tanabé, exposer avec lyrisme les affinités qui l'ont poussé, au sens quasiment religieux du terme, à se consacrer à Pascal. Il a fallu qu'un savant comme $\mathrm{M}$. Maeda repère que les variantes pascaliennes forment système, et s'impose au premier plan par sa méthode de double lecture - méthode dont nous avons eu à Tokyo, grâce à $M$. Sato, une saisissante illustration cinématographique: nous pouvons dire que nous avons vu la main de Pascal. Il a fallu enfin que, depuis plus de dix ans, les efforts des promoteurs du colloque soient soutenus et en quelque sorte cautionnés par l'ampleur des recherches pascaliennes menées dans les deux pays: le bilan des études récentes dressé, du côté français, par M. Sellier et, du côté nippon, par M. Nishikawa, révèle en effet que la production japonaise est presque aussi abondante que la nôtre, même si 10 \% seulement nous en sont linguistiquement accessibles. De ces deux puissants courants, notre colloque aura été la confluence.

Quel bilan tirer, pour conclure, de cette réunion qui, certes ne s'est pas contentée de tirer le bilan des années écoulées? Nous nous heurtions à un double obstacle : la distance de l'Orient à l'Occident, la distance du passé au présent. Tout me sépare de Pascal, disait T. Shiokawa, la langue, la culture, la morale et surtout la religion. Pourtant, comme ils ont appris la langue de Pascal, nos hôtes japonais ont aussi appris à connaitre sa culture, sa morale et sa religion - et ils nous ont aidés à mieux les connaître nous-mêmes. Toutes les dimensions de l'univers pascalien ont été visitées, éclairées de lumières jumelles: dimension textuelles, rhétorique, scientifique, politique, mystique - en même temps qu'une contribution inappréciable était apportée à l'étude de la réception de son œuvre. Et Port-Royal n'a pas été négligé, loin de là, puisqu'ont été scrutées la spiritualité de la 
Mère Agnès, la correspondance de la Mère Angélique, la personnalité de la Mère Angélique de Saint-Jean et que, dans la mouvance du monastère, nous nous sommes arrêtés sur Nicole, sur Philippe de Champaigne, saluant plus loin La Rochefoucauld, Du Vaucel, Quesnel. Nous avons pris mesure simultanément de la double universalité de notre champ d'études : il couvre tous les domaines et il touche tous les pays. On pourrait ajouter une troisième universalité, celle des temps. La distance passé-présent nous était commune avec les Japonais par rapport à Port-Royal, et c'est un Japonais - M. Izuka - qui nous a affermis dans la conviction que Port-Royal n'était pas anachronique mais délivrait un message urgent à la conscience morale et à la rationalité technologique de notre modernité. De cette distance chronologique comme des autres il faut dire qu'elle a été surmontée sans être abolie : notre intention n'était pas de concocter un concordisme à bon marché ni de prôner un retour archaïsant au siècle d'or du jansénisme. Le sentiment de la différence est le garde-fou de l'activité critique, comme celui de la sympathie en est le moteur. L'un et l'autre se sont donnés carrière au cours de ce colloque pour nous permettre d'atteindre ce que $T$. Shiokawa a excellemment défini comme l'équilibre entre la tolérance pratique et l'intransigeance théorique. Comme dans le procédé littéraire du dépaysement, où le détour par l'étranger permet de mieux se comprendre soi-même, notre meilleure compréhension du Japon a enrichi et approfondi notre regard sur nos propres valeurs culturelles : désormais, nous ne verrons plus Pascal et Port-Royal tout à fait avec les mêmes yeux.

Le 26 septembre, devant le Grand Boudha de Kama Kura

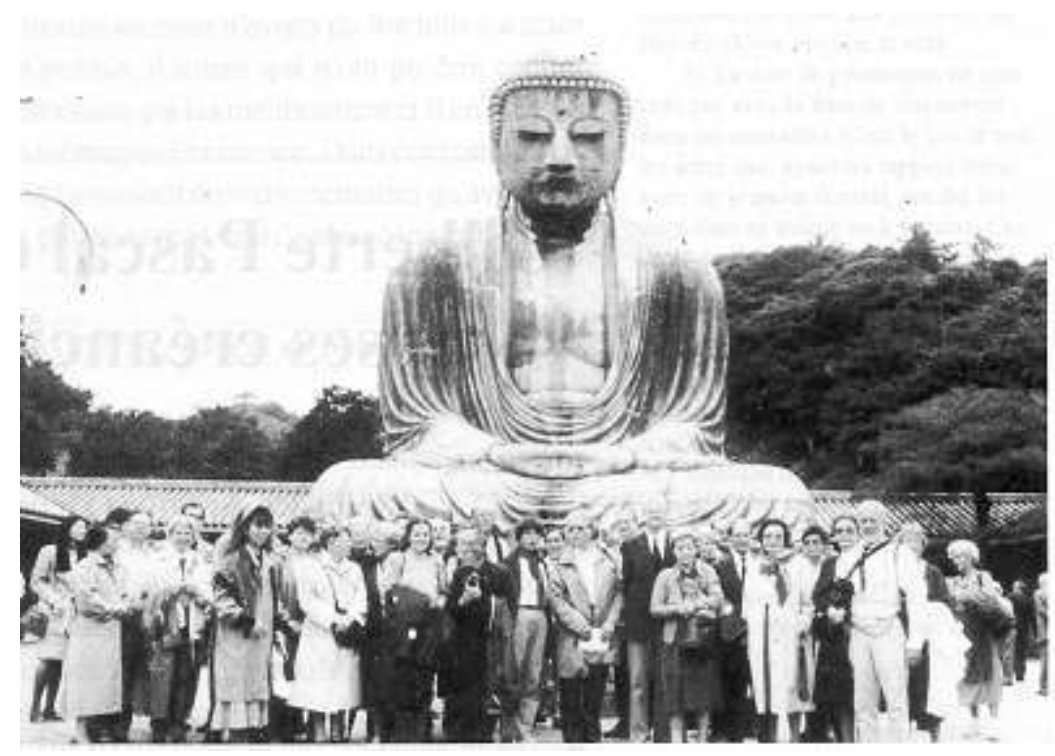

Cliché Genji Yasui 
Le 8 octobre, Sengari, la maison des séminaires

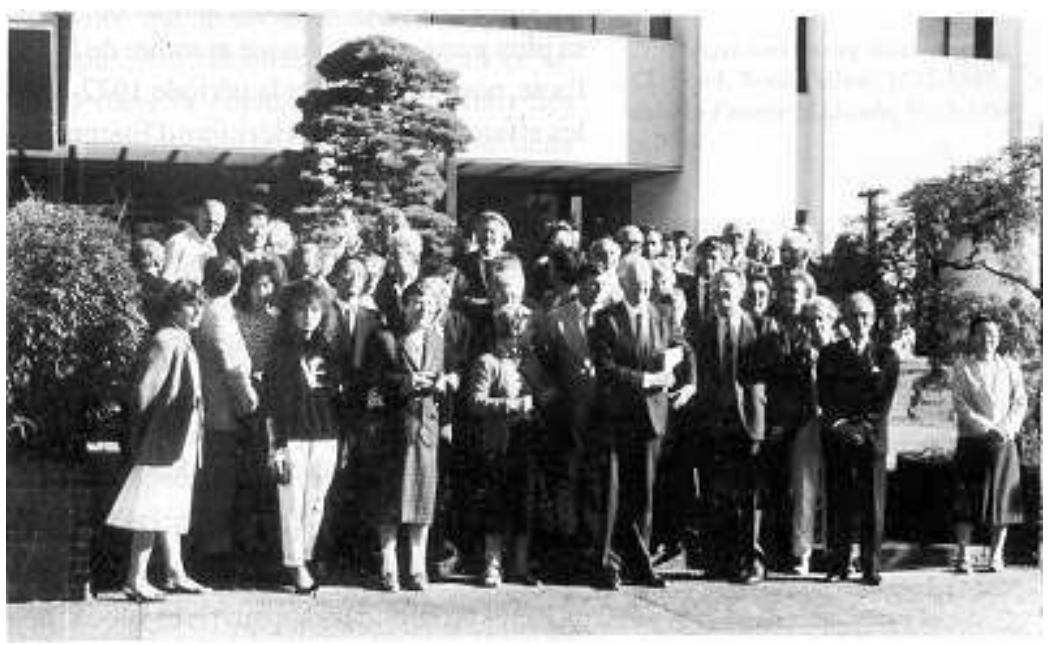

Cliché Madeleine Defrenne.

INDEX

Mots-clés : Pascal

Index géographique : Japon 